\title{
Celecoxib induces cell apoptosis coupled with up-regulation of the expression of VEGF by a mechanism involving ER stress in human colorectal cancer cells
}

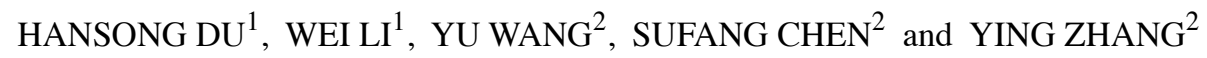 \\ Departments of ${ }^{1}$ Gastrointestinal Surgery, Union Hospital, and ${ }^{2}$ Biochemistry and Molecular Biology, \\ Tongji Medical College, Huazhong University of Science and Technology, Wuhan, Hubei 430030, P.R. China
}

Received March 11, 2011; Accepted April 18, 2011

DOI: 10.3892/or.2011.1297

\begin{abstract}
Increasing evidence suggests that celecoxib, a COX-2 inhibitor with potent anticancer activity exerts its effects not only through COX-2, but also through COX-2-independent mechanisms. In the present study, we hypothesized that endoplasmic reticulum stress (ERS) is involved in the up-regulation of VEGF expression induced by celecoxib in colorectal cancer HCT116 cells and that ERS is a major mechanism by which celecoxib triggers tumor cell death in a COX-2-independent manner. HCT116 cells, which do not express COX-2, were cultured in the absence or presence of celecoxib. VEGF expression was detected by quantitative real-time RT-PCR and Western blotting. ERS triggered by celecoxib was determined by expression of ER chaperones and other markers. PBA (an inhibitor of ERS) and GRP78 overexpression were both used to prevent ERS. Cell apoptosis was evaluated by TUNEL assay and fluorescence activated cell sorting. In HCT116 cells, celecoxib increased VEGF production with time-course and dose-response curves similar to those observed for the increase of the ER chaperone, GRP78. CHOP, a marker of ERS involved in apoptosis, was also increased by celecoxib. Moreover, celecoxib promoted cell apoptosis. Both apoptosis and up-regulation of VEGF were prevented by protecting cells
\end{abstract}

Correspondence to: Dr Ying Zhang, Department of Biochemistry and Molecular Biology, Tongji Medical College, Huazhong University of Science and Technology, 13 Hangkong Road, Wuhan, Hubei 430030, P.R. China

E-mail: seaside_ying@hotmail.com

Abbreviations: ATF6, activating transcription factor 6; ATF4, activating transcription factor 4 ; $\mathrm{CHOP}, \mathrm{C} / \mathrm{EBP}$ homologous transcription factor; COX-2, cyclooxygenase-2; ERS, endoplasmic reticulum stress; FACS, fluorescence activated cell sorting; GRP78, glucoseregulated protein 78 ; HNSCC, head and neck squamous cells carcinoma; IREI, inositol-requiring kinase I; PBA, 4-phenyl butyric acid; PERK, protein kinase R-like ER kinase; UPR, unfolded protein response; VEGF, vascular endothelial growth factor

Key words: celecoxib, VEGF, endoplasmic reticulum stress, cell apoptosis from ERS. Celecoxib induces cell apoptosis and up-regulation of VEGF in HCT116 cells via activation of the ERS response. Further studies are necessary to evaluate whether the combination of celecoxib with anti-VEGF agents is a promising therapeutic modality for cancer.

\section{Introduction}

Colorectal cancer is one of the most common malignancies worldwide, and, despite advances in chemotherapy, remains a major cause of cancer-related deaths. Available anticancer drugs for colorectal cancer are associated with substantial side effects (1). For these reasons, there is much interest in novel pharmacological therapies. Among different options, the roles played by the cyclooxygenase-2 (COX-2) pathway and gut hormones in the control of colorectal cancer growth represent areas of active investigation (2).

Celecoxib is a selective inhibitor of COX-2 and it has potent anticancer activity in various animal tumor models. This effect is particularly well documented in relationship to colon and rectal cancer (3-6). Although the effect of celecoxib was believed to be based solely on its inhibitory effects of COX-2 activity, additional pharmacological activities of celecoxib have been discovered and have challenged this notion. Interestingly, there are an increasing number of reports describing potent antiproliferative and proapoptotic effects of these drugs in the absence of any apparent involvement of COX-2 (7-11). One of several lines of evidence excluding the potential contribution of $\mathrm{COX}-2$ came from the use of COX-2 incompetent derivatives of celecoxib. Despite inability to inhibit COX-2, these derivatives faithfully mimic the anticancer actions of celecoxib in vitro and in vivo (12-14). In addition, celecoxib has been shown to inhibit growth of cell lines that are deficient for COX-2 expression $(15,16)$ and treatment with celecoxib results in growth inhibition of xenografts of COX-2deficient colorectal and prostate cancer cell lines in nude mice $(17,18)$. Therefore, the COX-2-independent mechanisms of celecoxib anti-cancer action are of considerable interest.

It has been frequently reported that COX-2 promotes angiogenesis through up-regulation of vascular endothelial growth factor (VEGF) production in a prostaglandin-dependent mechanism in several cancer cells, and it was anticipated that 
celecoxib would inhibit VEGF synthesis in a COX-2-dependent manner (19-21). However, we recently noted that celecoxib up-regulated the expression of VEGF in HCT116 cells (unpublished data). Since HCT116 cells do not express COX-2 because of promoter hypermethylation, this clearly suggests a non-COX-2 dependent activity. This unexpected, but interesting finding prompted us to look for the mechanism by which celecoxib up-regulated VEGF expression.

Recently, endoplasmic reticulum stress (ERS) has been noted as a major mechanism by which celecoxib can trigger tumor cell death in vitro and in vivo (10, 22-24). Furthermore, some ER stressors, such as amino acid deprivation and homocysteine, increase the expression of VEGF by activation of the unfolded protein response (UPR), a coordinated adaptive program in response to ERS (25). Hence, we hypothesized that ERS is involved in up-regulation of VEGF by celecoxib in HCT116 cells, since the celecoxib is a potential trigger of ERS in many cells. In addition, although studies indicate that celecoxib-induced ERS contributes to its apoptotic effect in some cancers $(10,11,22-24)$, this has not to date been documented in human colorectal cancer cells.

In this study, we demonstrate that celecoxib up-regulates VEGF expression by a mechanism involving ERS and confirm that celecoxib-induced ERS exerts apoptotic effects in HCT116 cells.

\section{Materials and methods}

Cell culture and treatment conditions. HCT116 human colon cancer cells were obtained from the American Type Culture Collection (ATCC; Manassas, VA, USA) and stored in our laboratory. Cells were cultured in McCoy's medium (Invitrogen, USA), with $10 \%$ fetal bovine serum (FBS) and supplemented with penicillin and streptomycin (both at 2\%). Cells were maintained in a humidified incubator at $37^{\circ} \mathrm{C}$, in the presence of $5 \% \mathrm{CO}_{2}$ and were subcultured following enzymatic digestion by trypsin/EDTA. Celecoxib was obtained as capsules from the pharmacy (Pfizer, USA), dissolved in DMSO at $100 \mathrm{mmol} / \mathrm{l}$ (stock solution, $-20^{\circ} \mathrm{C}$ ) and added to the cell culture medium in a manner that kept the final concentration of solvent below $0.1 \%$. Cells were exposed to celecoxib by changing the medium. Unless otherwise noted, cells $\left(1.0 \times 10^{4}\right.$ cells/well in 24-well plates, $6 \times 10^{4}$ cells/well in 6 -well plates, $6 \times 10^{5}$ cells in $100-\mathrm{mm}$ plates) were cultured for $24 \mathrm{~h}$ before use in experiments.

Real time RT-PCR analysis. Total RNA was extracted from cells using an RNeasy kit (Qiagen, German), according to the manufacturer's protocols. The concentration and purity of RNA was determined using a spectrophotometer. cDNA was synthesized with the MMLV reverse transcriptase (Promega, Madison, WI, USA). Synthesized cDNA was subjected to real-time RT-PCR (ABI PRISM 7700) using the SYBR-Green Real-Time PCR Master mix (Applied Biosystems, USA) and analyzed with the ABI PRISM 7700 Sequence Detection Software according to the manufacturer's instructions. Primers and conditions were used as previously described (38). Specificity was confirmed by electrophoretic analysis of the reaction products and by inclusion of template- or reverse transcriptionfree controls. To normalize the amount of total RNA present in each reaction, the actin gene was used as an internal control.
Werstern blot analysis. Cells were lysed on ice with lysis buffer containing $1 \mathrm{mM}$ phenylmethylsulfonyl fluoride, $1 \mu \mathrm{g} / \mathrm{ml}$ leupeptin and $2 \mu \mathrm{g} / \mathrm{ml}$ aprotinin. Lysates were centrifuged for $10 \mathrm{~min}$ at $4^{\circ} \mathrm{C}$ and at $14,000 \mathrm{rpm}$ to pellet cell debris. Protein concentrations were determined using an assay kit (Bio-Rad, Hercules, CA, USA). Supernatants were separated on $10 \%$ SDS-PAGE gels and transferred onto polyvinylidene fluoride membranes. Membranes were incubated in blocking buffer [tris-buffered saline (TBS), 0.1\% Tween-20, and 5\% non-fat dry milk) for $1 \mathrm{~h}$ at room temperature, followed by hybridization with anti-COX-2, anti-VEGF, anti-CHOP (all from Santa Cruz Biotechnology, CA, USA), anti-GRP78 antibody (from BD Transduction Laboratories, NJ, USA), or anti $\beta$-actin antibodies (Lab Vision, Fremont, CA, USA) at $4^{\circ} \mathrm{C}$ overnight. After 3 washes in TBS $/ 0.1 \%$ Tween-20, the membranes underwent hybridization with a horseradish peroxidase-conjugated secondary antibody (Life Technologies Inc.) for $1 \mathrm{~h}$ at room temperature, and were developed using the ECL reagent (Pierce Chemical Co., Rockford, IL, USA).

Establishment of stable T24/83 cell lines overexpressing GRP78. HCT116 cells grown to $30 \%$ confluence were transfected with $5 \mu \mathrm{g}$ of the pcDNA3.1-GRP78 expression plasmid (a gift from Dr Austin Richard, McMaster University, Canada) using $30 \mu 1$ of SuperFect transfection reagent (Qiagen), as described by the manufacturer. As a vector control, HCT116 cells were transfected with pcDNA3.1 (Invitrogen) under the same conditions. Stable transfectants were selected in complete medium containing $1.2 \mathrm{mg} / \mathrm{ml}$ G418 (Life Technologies Inc.) for 2 weeks. G418-resistant clones were subsequently isolated and cultured in complete medium containing $1.0 \mathrm{mg} / \mathrm{ml} \mathrm{G} 418$. Overexpression of GRP78 was assessed using Western blotting.

Analysis of apoptosis by TUNEL. Fragmented DNA was detected by TUNEL assay (Boehringer Mannheim, German) according to the manufacturer's protocols. For quantitative evaluation of apoptotic cells, 20 fields (at least 200 cells) were counted in each preparation. The apoptosis rate (\%) was calculated as follows: (number of apoptotic cells)/(number of total cells) $\mathrm{x} 100 \%$.

Analysis of apoptosis by fluorescence activated cell sorting $(F A C S)$. Apoptosis was monitored by FACS analysis as previously described (26). Briefly, cells were cultured in $100-\mathrm{mm}$ plates and collected by centrifugation. Pellets were fixed with $70 \%$ ethanol for $4 \mathrm{~h}$ at $-20^{\circ} \mathrm{C}$ and centrifuged again. Pellets were resuspended in phosphate-citrate buffer $(0.2 \mathrm{M}$ $\mathrm{Na}_{2} \mathrm{HPO}_{4}$ and $4 \mathrm{mM}$ citric acid) and incubated for $20 \mathrm{~min}$ at room temperature. After centrifugation, the pellets were resuspended in DNA staining solution $(50 \mathrm{mg} / \mathrm{ml}$ PI and $10 \mathrm{mg} / \mathrm{ml}$ RNase A) and incubated for $20 \mathrm{~min}$ at RT. Samples were scanned with a FACSCanto II (BD Biosciences, NJ, USA) cell sorter and the percentage of apoptotic cells was determined.

Statistical analysis. All assays were conducted 3 times and found to be reproducible. Data were expressed as mean \pm SD. and analyzed by SPSS 10.0 software (SPSS, Chicago, IL). Differences were considered statistically significant at $\mathrm{p}<0.01$. 
$\mathbf{A}$

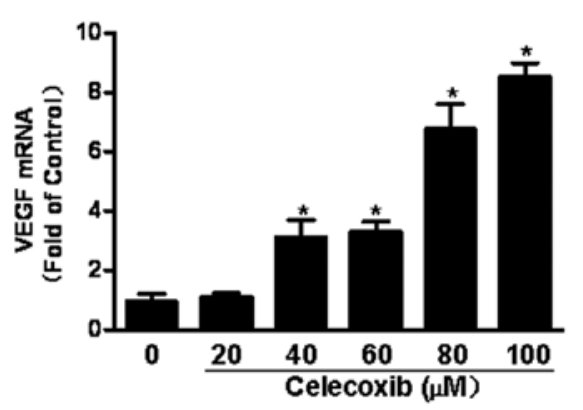

C
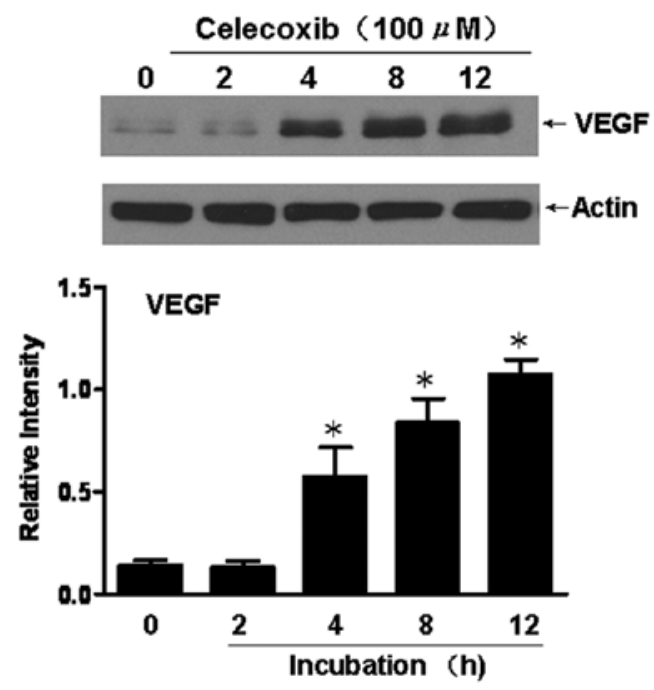

B
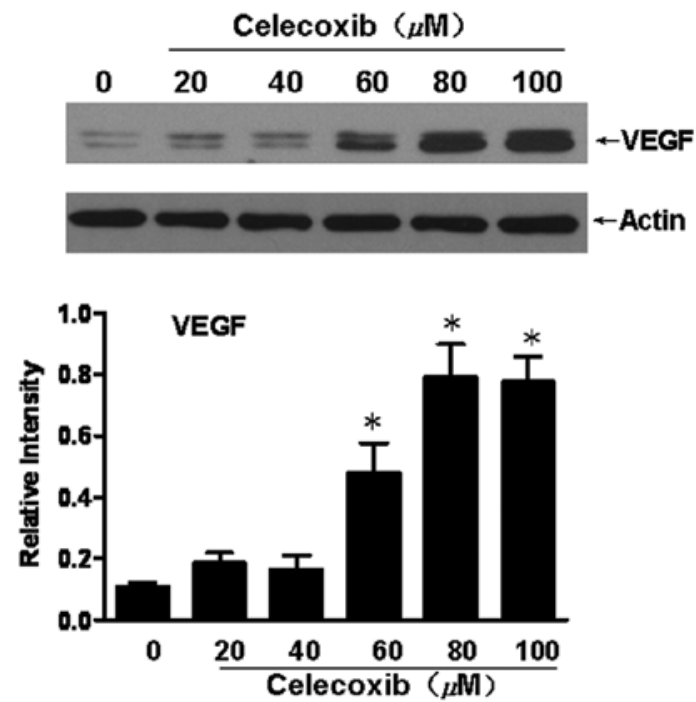

D

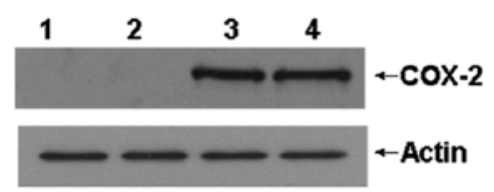

Figure 1. Up-regulation of VEGF by celecoxib. (A) HCT116 cells were incubated with the indicated concentrations of celecoxib for $12 \mathrm{~h}$ and total RNA was extracted. Samples were subjected to real-time RT-PCR by use of a specific primer for VEGF. (B) Cells were incubated with the indicated concentrations of celecoxib for $12 \mathrm{~h}$. Supernatants of whole cell extracts were analyzed by Western blotting with an antibody against VEGF or actin. (C) HCT116 cells were incubated with $100 \mu \mathrm{M}$ celecoxib for the indicated time periods and Western blotting used to analyze the expression of VEGF. (D) Western blot analysis of COX-2 expression in HCT116 cells. Lanes 1 and 2, samples from HCT116 cells; lanes 3 and 4, positive controls of COX-2 expression. Values of real-time RT-PCR or Western blotting were normalized to actin gene expression and expressed relative to the control sample (i.e. without celecoxib) or as relative intensity ( ${ }^{*} \mathrm{p}<0.01$ control vs. others).

\section{Results}

Celecoxib up-regulates the expression of VEGF in HCT116 cells. HCT116 cells were cultured without or with celecoxib as indicated in Fig. 1. Real-time PCR and immunoblotting techniques were used to assess VEGF. As shown in Fig. 1A and $\mathrm{B}$, celecoxib clearly increased mRNA levels and cellular protein levels of VEGF at doses of 40 and $60 \mu \mathrm{M}$, respectively. A time course study further demonstrated a VEGF protein increase occurring after treatment with $100 \mu \mathrm{M}$ celecoxib for $4 \mathrm{~h}$ with further increases until $12 \mathrm{~h}$ (Fig. 1C). It has been reported that HCT116 cells do not express COX-2 because of promoter hypermethylation. We confirmed this observation by Western blotting and found no detectable COX-2 expression in HCT116 cells (Fig. 1D). These results show that the COX-2selective inhibitor, celecoxib, up-regulates the expression of VEGF at the mRNA and protein level independently of COX-2 inhibition.
Mechanism for up-regulation of VEGF by celecoxib. To test our hypothesis that ERS is involved in up-regulation of VEGF by celecoxib in HCT116 cells, cells were treated with increasing concentrations of celecoxib or with $100 \mu \mathrm{M}$ celecoxib for 2,4 , 8 or 12 h. Western blotting was used to detect the expression of the ERS marker glucose-regulated protein 78 (GRP78) and another ERS response protein, C/EBP homologous transcription factor (CHOP). CHOP is a proapoptotic factor and is important to the induction of apoptosis by ER stressors $(27,28)$. As shown in Fig. 2, celecoxib increased the expression of GRP78 and CHOP. GRP78 expression increased after treatment with $100 \mu \mathrm{M}$ celecoxib for $4 \mathrm{~h}$, while $\mathrm{CHOP}$ expression increased after $8 \mathrm{~h}$, indicating that celecoxib triggers ERS in HCT116 cells. In addition, the time course and dose-response curves for the up-regulation of GRP78 were similar to those observed for the celecoxib-induced VEGF increase (Figs. 1 and 2).

Next, we sought to determine whether celecoxib-induced ERS leads to up-regulation of VEGF expression. Overexpression 
A
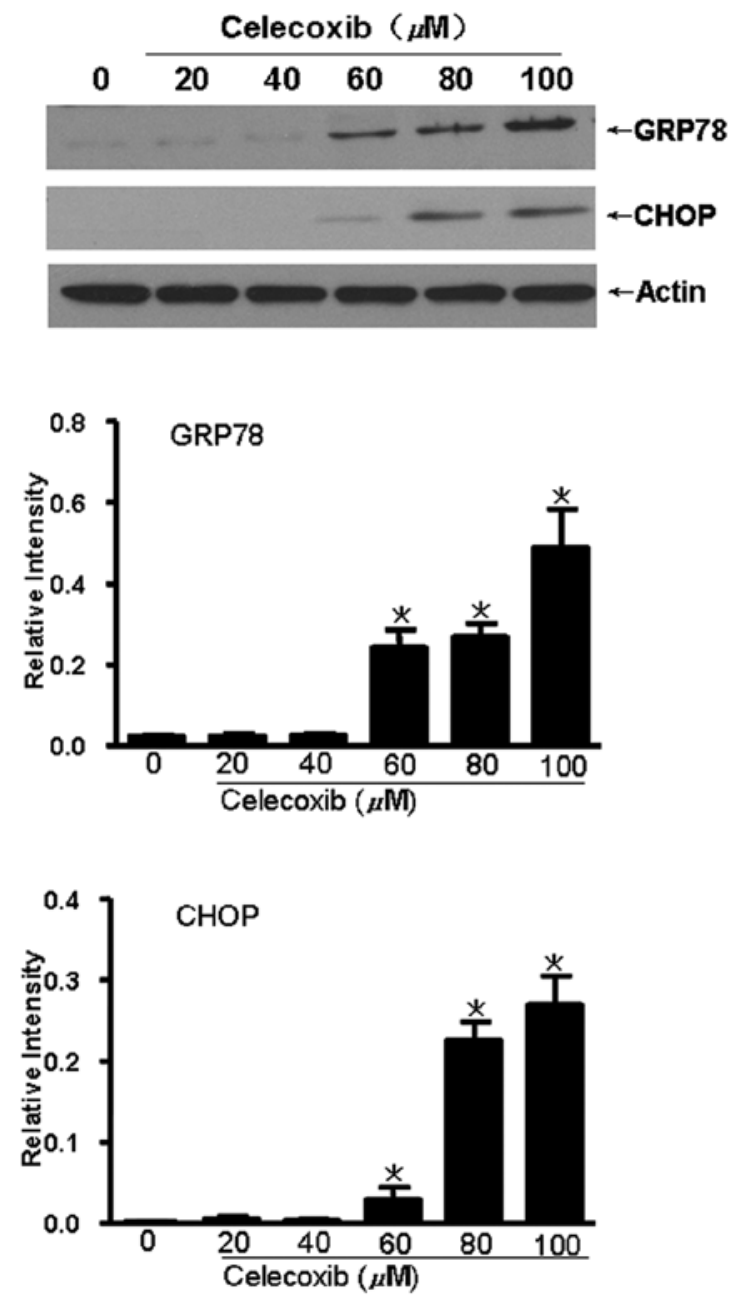

B
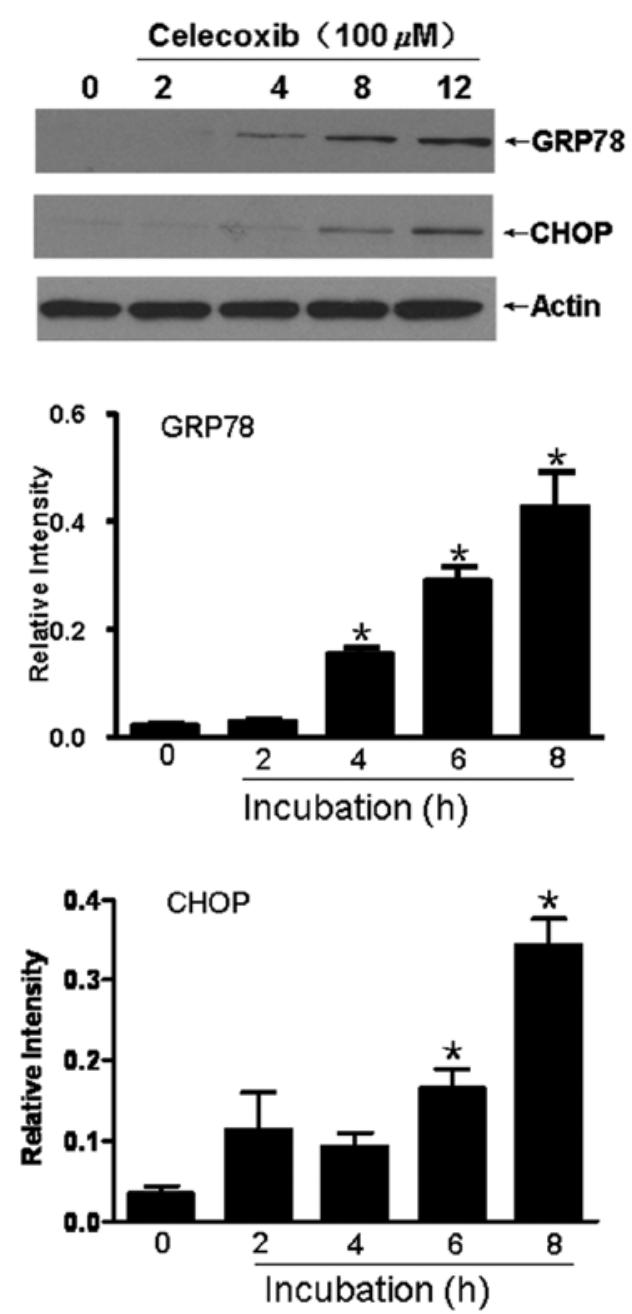

Figure 2. Celecoxib induces the expression of GRP78 and CHOP in HCT116 cells. (A) Western blot analysis of protein levels of GRP78 and CHOP in HCT116 cells cultured for $12 \mathrm{~h}$ in the absence (control) or presence of celecoxib in indicated concentrations. (B) Western blot analysis of protein levels of GRP78 and CHOP in HCT116 cells cultured in the absence or presence of $100 \mu \mathrm{M}$ celecoxib for the indicated time periods. Total protein lysates were separated on $10 \%$ SDS-PAGE and transferred to nitrocellulose membranes and immunostained with antibodies against GRP78, CHOP or actin. Values were normalized to actin and expressed as relative intensity ( $\mathrm{p}<0.01$ control vs. others).

of GRP78 protects cells from ERS and restores ER homeostasis (29-31). Stably transfected cells overexpressing GRP78 (HCT116-GRP78 cells) were generated to assess whether blocking celecoxib-induced ERS suppressed the expression of VEGF. As shown in Fig. 3A, HCT116-GRP78 cells exhibited a 6.2-fold increase in GRP78 protein levels, compared with wild-type or vector-transfected cells, indicating that HCT116 cells overexpressing GRP78 were successfully established. Further, when treated with celecoxib, the expression of VEGF was greatly increased in vector-transfected cells after celecoxib treatment for $4 \mathrm{~h}$, but in HCT116-GRP78 cells, this up-regulation was clearly blocked (Fig. 3B). The chemical chaperone 4-phenyl butyric acid (PBA), a specific inhibitor of ERS was also used to test the effect of ERS induced by celecoxib on the expression of VEGF. PBA suppressed the occurrence of ERS induced by celecoxib efficiently (Fig. 4). Furthermore, in cells treated with PBA, the induction of VEGF protein by celecoxib was greatly attenuated (Fig. 4).

Taken together, these results indicate that the ERS response plays an important role in the up-regulation of VEGF induced by celecoxib.
The effect of celecoxib-induced ERS on cell growth. Since celecoxib triggers ERS and CHOP induction in HCT116 cells, it is plausible that celecoxib-induced ERS may cause apoptosis in these cells. We thus investigated the apoptosis in different HCT116 cells (HCT116; HCT116-pcDNA; HCT116-GRP78) in the presence or absence of celecoxib. After treatment with celecoxib $(100 \mu \mathrm{M})$ for $24 \mathrm{~h}$, TUNEL staining showed more apoptosis in HCT116 or HCT116-pcDNA cells compared with their untreated controls. Conversely, in HCT116-GRP78 cells, celecoxib did not increase apoptosis (Fig. 5). FACS was also used to evaluate cell apoptosis mediated by celecoxib-induced ERS. The results showed that a marked sub-G0/G1 peak appeared in the presence of celecoxib, but this peak was diminished by PBA (Fig. 6). We thus demonstrate that celecoxib promotes apoptosis in HCT116 cells and this proapoptotic effect is due to celecoxib-induced ERS.

\section{Discussion}

The present study provides the first evidence that celecoxib up-regulates VEGF expression by inducing ERS. We further 
A
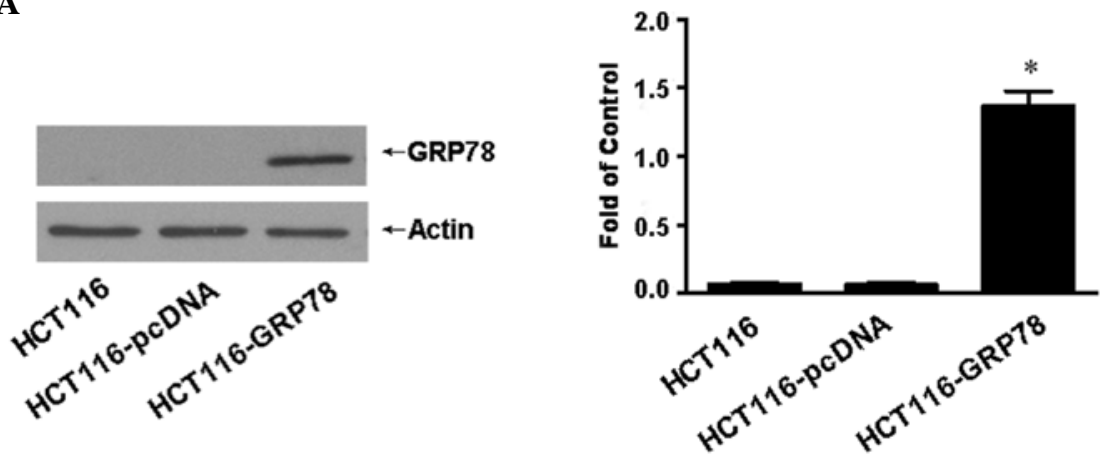

B
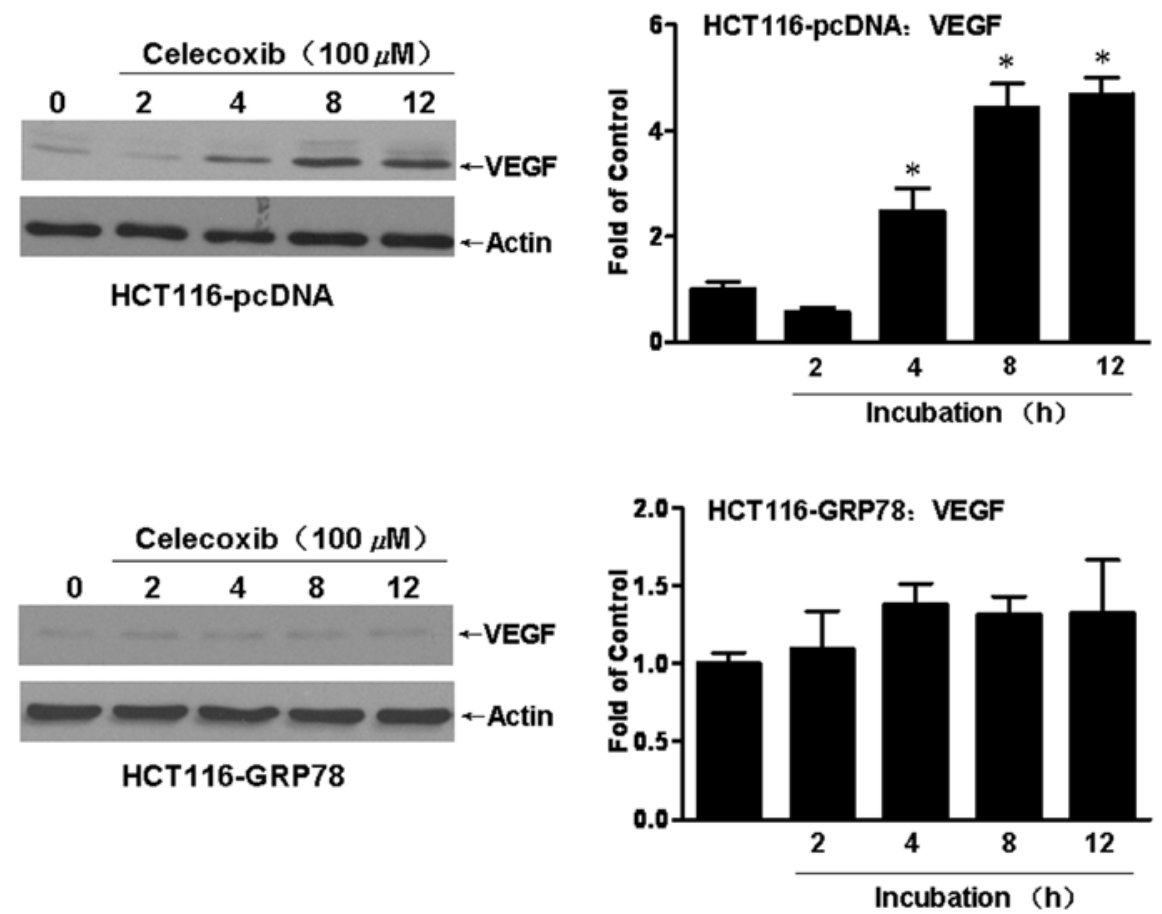

Figure 3. Overexpression of GRP78 prevents the increase in protein levels of VEGF. (A) Western blot analysis of GRP78 expression in wild-type (HCT116), vector transfected (HCT116-pcDNA), or GRP78 overexpressing (HCT116-GRP78) cells ("p<0.01 HCT116-GRP78 vs. HCT116 or HCT116-pcDNA). (B) Western blot analysis of protein levels of VEGF in vector transfected (HCT116-pcDNA) and GRP78 overexpressing (HCT116-GRP78) cells in the absence of presence or $100 \mu \mathrm{M}$ celecoxib for the indicated time periods. Total protein was extracted, and Western blot analysis was performed with antibodies against GRP78, VEGF and actin. Values were normalized to actin and expressed relative to the control ("p $<0.01$ control vs. others).

confirmed that in the COX-2-deficient colorectal cancer line HCT116, ERS plays a key role in celecoxib-induced apoptosis.

The critical contribution of the ERS to tumor cell growth and survival has only recently begun to be recognized (32-34). The primary purpose of the ERS response is to alleviate the disturbance and restore proper ER homeostasis; however, in the case of intense or persistent ERS, these pathways will trigger programmed cell death/apoptosis. One of the central prosurvival regulators of the ERS response is GRP78, which has an important role in protein folding and assembly, in targeting misfolded protein for degradation, and in controlling the activation of transmembrane ERS sensors. It is, therefore, considered to be the paradigmatic marker of the ERS response. On the other hand, CHOP is a critical executioner of the proapoptotic arm of ERS. In cases of intense or persistently high stress, the defensive functions (such as elevated levels of GRP78) are overwhelmed, and the proapoptotic components (such as CHOP) will gain dominance and trigger cell death. Overexpression of GRP78 can protect cells from ERS and help restore ER homeostasis (29-31). We demonstrated that celecoxib-induced ERS increased the expression of GRP78 and CHOP, and induced cell apoptosis in HCT116 cells. Furthermore, apoptosis was reversed by overexpression of GRP78 or by the ERS inhibitor, PBA. These results indicate that, in the human colorectal cancer line HCT116, celecoxib exerted its proapoptotic functions by triggering the ERS. Our results in HCT116 cells together with previous and recent new findings in other cells $(11,22-24)$ suggest that celecoxib triggers ERS as a part of its general mechanism of action, independent of cell types, and this induction may be a key mechanism of the anti-cancer activity of celecoxib.

Angiogenesis is necessary for tumors to grow beyond a certain size, and it is a prerequisite for tumor invasion and metastasis. A relationship between COX-2 and angiogenesis has been noted, and hence it is considered that COX-2 inhibitors may be effective as antiangiogenic agents (19-21). However, in this study, exposure of HCT116 cells to celecoxib led to up-regulation of VEGF expression. VEGF has been shown to 
A
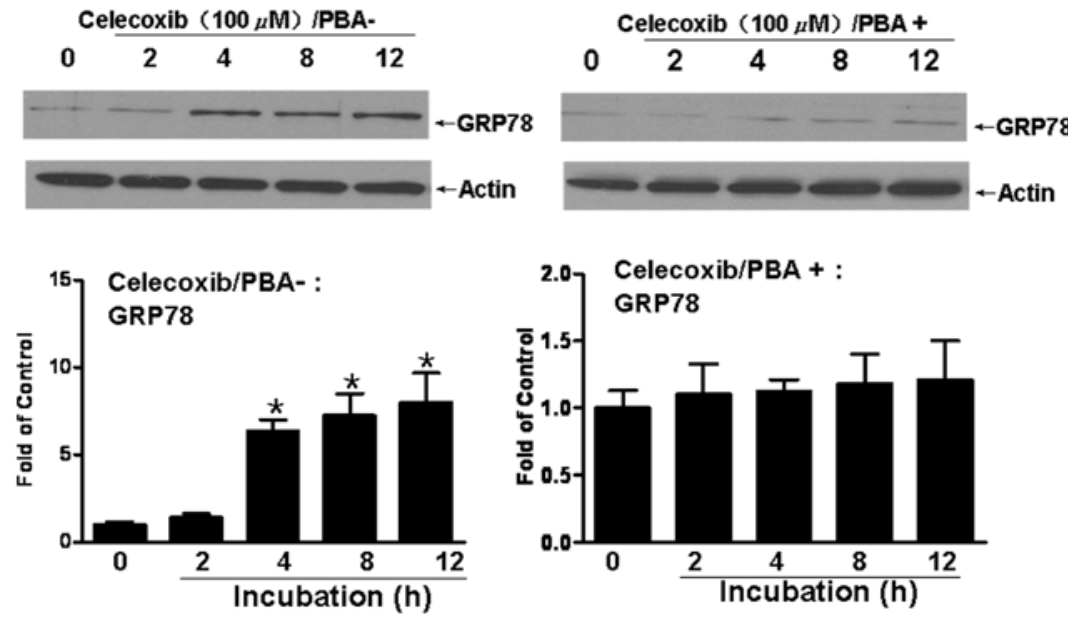

B
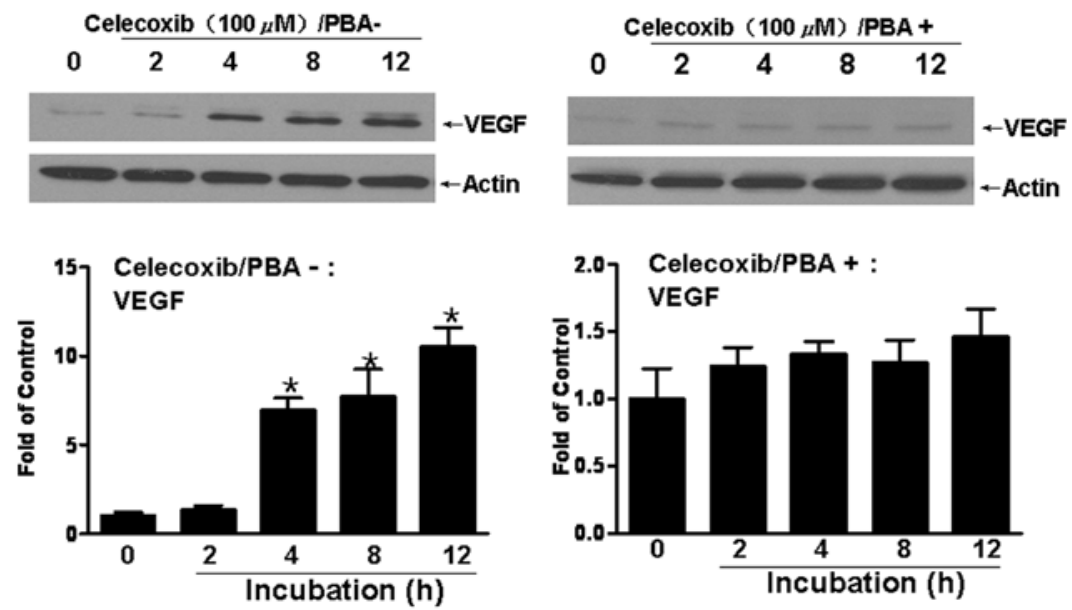

Figure 4. Protection of cells from ERS by PBA prevents the increase in protein levels of VEGF. HCT116 cells were incubated with $100 \mu \mathrm{M}$ celecoxib for the indicated time periods. Before celecoxib treatment, cells were pretreated without or with $3 \mathrm{mM}$ PBA for $6 \mathrm{~h}$. (A) Western blot analysis of the effect of PBA on the expression of GRP78 induced by celecoxib. (B) Western blot analysis of VEGF when ERS is suppressed by PBA. Total protein was extracted, and Western blot analysis was performed with antibodies against GRP78, VEGF and actin. Values were normalized to actin and expressed relative to control ("p $<0.01$ control vs. others).

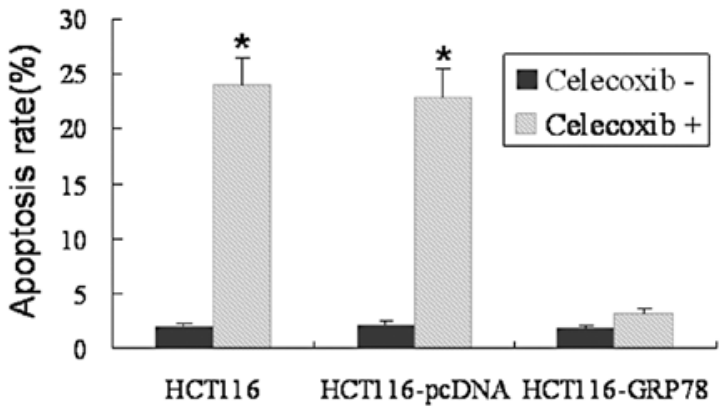

Figure 5. Celecoxib induces cell apoptosis which is prevented by overexpression of GRP78. Cells (HCT116, HCT116-pcDNA or HCT116-GRP78 cells) were either untreated or treated with $100 \mu \mathrm{m}$ celecoxib for $24 \mathrm{~h}$, and apoptosis was determined by TUNEL ( $\mathrm{p}<0.01$ celecoxib+ vs. celecoxib-).

be the major mediator of physiological and pathological angiogenesis. In addition, recent studies suggest that VEGF is associated with cell growth and anti-apoptosis. Castro-Rivera et al (35) found that VEGF could suppress apoptosis in lung and breast cancer cells. Pidgeon et al (36) found that VEGF could up-regulate Bcl-2 levels in breast cancer cells, thus inhibiting apoptosis in both tumor cells and vascular endothelial cells. The up-regulation of VEGF expression by celecoxib has also been observed in head and neck squamous cell carcinoma (HNSCC) cells (37). Park et al (37) observed that COX-2 inhibitors increase VEGF synthesis, but that COX-2-siRNA inhibited VEGF production in HNSCC. Rather, small molecule COX-2 inhibitors increased VEGF synthesis irrespective of inhibition of prostaglandins production. Taken together, these results indicate that: i) up-regulation of VEGF expression by COX-2 inhibitors, at least by celecoxib, is not a unique phenomenon observed only in the COX-2-deficient colorectal cancer line HCT116; ii) this up-regulation of VEGF by celecoxib is an off-target, COX-2-independent action, and this action may overwhelm the effect of VEGF down-regulation due to inhibition of COX-2 activity. In addition, Park et al found that celecoxib in the range of doses that increased VEGF production could inhibit cell proliferation (37). These data are in agreement to our results indicating that celecoxib promotes cell apoptosis coupled with up-regulation of VEGF expression.

The mechanisms whereby celecoxib increases VEGF production were elucidated in the present study. In cells 
A

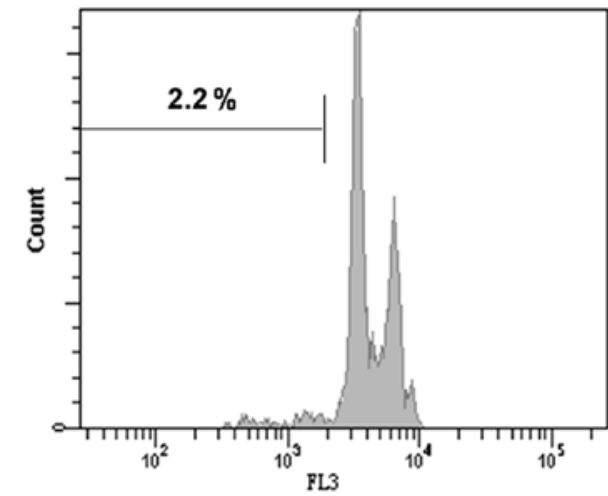

C

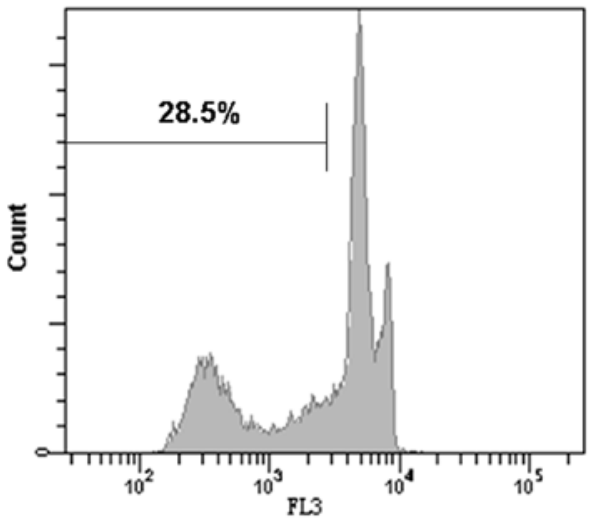

B

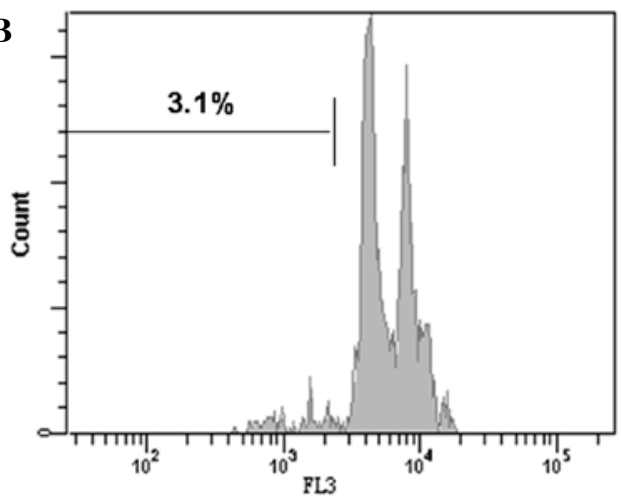

D

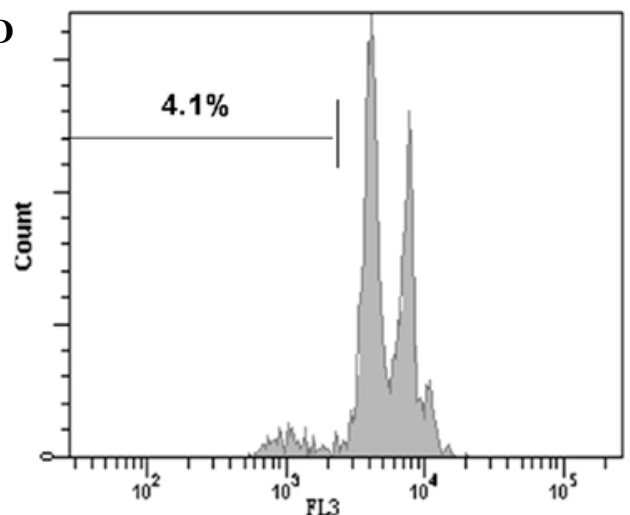

Figure 6. PBA protects cells from celecoxib-induced apoptosis. HCT116 cells were pretreated without or with PBA 3 mM for 6 h, and then incubated in the presence or absence of $100 \mu \mathrm{M}$ celecoxib for $24 \mathrm{~h}$. Cells were stained with propidium iodide, followed by detection of apoptotic cells using FACS. Cells with sub-G0/G1 DNA content are apoptotic, and the percentage of total cells in this area is given. (A) Cells exposed to neither PBA nor celecoxib; (B) cells exposed to PBA but not to celecoxib; (C) cells exposed to celecoxib but not to PBA; (D) cells exposed to both PBA and celecoxib.

overexpressing GRP78 (HCT116-GRP78), celecoxib mediated VEGF expression was blocked, implicating induction of ERS in the process. The results indicate that ERS is involved in the up-regulation of VEGF induced by celecoxib. This view is further supported by the observation that VEGF expression was not increased in response to celecoxib in the presence of PBA, an inhibitor of ERS, in HCT116 cells. Furthermore, from our data, it can be concluded that celecoxib induces cell apoptosis and up-regulates VEGF expression by the same mechanism (ERS) and this provides an explanation whereby celecoxib, in the range of doses that increases VEGF production, inhibits cell proliferation.

It is well known that the UPR, in response to ERS, is initiated by three distinct signaling branches, protein kinase R-like ER kinase (PERK), inositol-requiring kinase I (IREI), and activating transcription factor 6 (ATF6) (28). We did not study which branches or which downstream components were involved in the up-regulation of VEGF by celecoxib in detail, but previous study has shown the transcription factor ATF4, a downstream effector of PERK, plays a key role in up-regulating VEGF expression by homocysteine (25). In addition, it has been reported that the PERK-eIF2 $\alpha$-ATF4 pathway is activated by celecoxib in human gastric cells (10). Therefore, it is speculated that ATF4 might be important for the celecoxibinduced up-regulation of VEGF expression in HCT116 cells.

Some authors have reported inhibition of VEGF production by NSAIDs in colon cancer cells. Wang et al (39) found that celecoxib significantly reduced the levels of PGE2 and VEGF in xenografts of HT-29 cells. Compared to their study, the HCT116 cells used in this study are COX-2 negative; therefore, celecoxib exerts a COX-2 independent effect and thus would not decrease VEGF production via the prostaglandin-dependent mechanism. Abdelrahim and Safe (40) reported that celecoxib decreased VEGF production in colon cancer cells expressing and not expressing COX-2, but they used a low concentration of celecoxib $(30 \mu \mathrm{M})$. It is postulated that only high-dose celecoxib triggers the ERS response and therefore up-regulates VEGF production (in our study, celecoxib triggered ERS at doses of $60 \mu \mathrm{M}$ or higher). In fact celecoxib has been used at $800 \mathrm{mg} /$ day in clinical oncology trials, twice the maximum anti-inflammatory dose.

In conclusion, celecoxib at relatively high doses induces cell apoptosis and up-regulates VEGF expression in HCT116 cells. Both of the two functions arise from the induction of ERS by celecoxib. VEGF may attenuate the anti-cancer action of celecoxib because of its potential to induce angiogenesis and thus, the activity of celecoxib combined with other drugs targeted to VEGF should be studied.

\section{Acknowledgements}

We thank Dr Austin (McMaster University) for the pcDNA3.1/ GRP78 and Dr Ingram (McMaster University) for editing the manuscript. This study was funded by grants 30700296 of the Natural Science Foundation of The People's Republic of China and 200850731355 of the Chenguang project of Wuhan. 


\section{References}

1. Kelly H and Goldberg RM: Systemic therapy for metastatic colorectal cancer: current options, current evidence. J Clin Oncol 23: 4553-4560, 2005

2. Brown JR and DuBois RN: COX-2: a molecular target for colorectal cancer prevention. J Clin Oncol 23: 2840-2855, 2005.

3. Sheng H, Shao J, Kirkland SC, et al: Inhibition of human colon cancer cell growth by selective inhibition of cyclooxygenase-2. J Clin Invest 99: 2254-2259, 1997.

4. Dannenberg AJ and Subbaramaiah K: Targeting cyclooxygenase-2 in human neoplasia: rationale and promise. Cancer Cell 4 431-436, 2003

5. Koehne $\mathrm{CH}$ and Dubois RN: COX-2 inhibition and colorectal cancer. Semin Oncol 31: 12-21, 2004.

6. Sørensen HT, Friis S, Nørgård B, et al: Risk of cancer in a large cohort of nonaspirin NSAID users: a population-based study. $\mathrm{Br}$ J Cancer 88: 1687-1692, 2003.

7. Arico S, Pattingre S, Bauvy C, et al: Celecoxib induces apoptosis by inhibiting 3-phosphoinositide-dependent protein kinase-1 activity in the human colon cancer HT-29 cell line. J Biol Chem 277: 27613-27621, 2002.

8. Kardosh A, Blumenthal M, Wang WJ, Chen TC and Schönthal AH: Differential effects of selective COX-2 inhibitors on cell cycle regulation and proliferation of glioblastoma cell lines. Cancer Biol Ther 3: 9-16, 2004.

9. Tegeder I, Pfeilschifter J and Geisslinger G: Cyclooxygenaseindependent actions of cyclooxygenase inhibitors. FASEB J 15:2057-2072, 2001.

10. Tsutsumi S, Namba T, Tanaka KI, et al: Celecoxib upregulates endoplasmic reticulum chaperones that inhibit celecoxib-induced apoptosis in human gastric cells. Oncogene 25: 1018-1029, 2006.

11. Chen ST, Thomas S, Gaffney KJ, Louie SG, Petasis NA and Schönthal AH: Cytotoxic effects of celecoxib on Raji lymphoma cells correlate with aggravated endoplasmic reticulum stress but not with inhibition of cyclooxygenase-2. Leuk Res 34: 250-253, 2010.

12. Kardosh A, Soriano N, Liu YT, et al: Multitarget inhibition of drug-resistant multiple myeloma cell lines by dimethyl-celecoxib (DMC), a non-COX-2 inhibitory analog of celecoxib. Blood 106 : 4330-4338, 2005.

13. Ding H, Han C, Zhu J, Chen CS and D'Ambrosio SM: Celecoxib derivatives induce apoptosis via the disruption of mitochondrial membrane potential and activation of caspase 9. Int J Cancer 113: 803-810, 2005.

14. Zhu J, Huang JW, Tseng PH, et al: From the cyclooxygenase-2 inhibitor celecoxib to a novel class of 3-phosphoinositidedependent protein kinase-1 inhibitors. Cancer Res 64: 4309-4318, 2004.

15. Maier TJ, Schilling K, Schmidt R, Geisslinger G and Grösch S: Cyclooxygenase-2(COX-2)-dependent and -independent anticarcinogenic effects of celecoxib in human colon carcinoma cells. Biochem Pharmacol 67: 1469-1478, 2001.

16. Waskewich C, Blumenthal RD, Li H, Stein R, Goldenberg DM and Burton J: Celecoxib exhibits the greatest potency amongst cyclooxygenase (COX) inhibitors for growth inhibition of COX-2-negative hematopoietic and epithelial cell lines. Cancer Res 62: 2029-2033, 2002.

17. Grosch S, Tegeder I, Niederberger E, Bräutigam L and Geisslinger G: COX-2 independent induction of cell cycle arrest and apoptosis in colon cancer cells by the selective COX-2 inhibitor celecoxib. FASEB J 15: 2742-2744, 2001.

18. Patel MI, Subbaramaiah K, Du B, et al: Celecoxib inhibits prostate cancer growth: evidence of a cyclooxygenase-2independent mechanism. Clin Cancer Res 11: 1999-2007, 2005.

19. Zhi YH, Liu RS, Song MM, et al: Cyclooxygenase-2 promotes angiogenesis by increasing vascular endothelial growth factor and predicts prognosis in gallbladder carcinoma. World J Gastroenterol 11: 3724-3728, 2005.

20. Liao Z, Mason KA and Milas L: Cyclo-oxygenase-2 and its inhibition in cancer: is there a role? Drugs 67: 821-845, 2007.

21. Yanni SE, McCollum GW and Penn JS: Genetic deletion of COX-2 diminishes VEGF production in mouse retinal Müller cells. Exp Eye Res 91: 34-41, 2010.
22. Tsutsumi S, Gotoh T, Tomisato W, et al: Endoplasmic reticulum stress response is involved in non-steroidal anti-inflammatory drug-induced apoptosis. Cell Death Differ 11: 1009-1016, 2004.

23. Cho HY, Thomas S, Golden EB, et al: Enhanced killing of chemo-resistant breast cancer cells via controlled aggravation of ER stress. Cancer Lett 282: 87-97, 2009.

24. Kardosh A, Golden EB, Pyrko P, et al: Aggravated endoplasmic reticulum stress as a basis for enhanced glioblastoma cell killing by bortezomib in combination with celecoxib or its non-coxib analogue, 2,5-dimethyl-celecoxib. Cancer Res 68: 843-851, 2008.

25. Roybal CN, Yang S, Sun CW, et al: Homocysteine increases the expression of vascular endothelial growth factor by a mechanism involving endoplasmic reticulum stress and transcription factor ATF4. J Biol Chem 279: 14844-14852, 2004.

26. Alves da Costa C, Paitel E, Mattson MP, et al: Wild-type and mutated presenilins 2 trigger p53-dependent apoptosis and down-regulate presenilin 1 expression in HEK293 human cells and in murine neurons. Proc Natl Acad Sci USA 99: 4043-4048, 2002.

27. Kaufman RJ: Orchestrating the unfolded protein response in health and disease. J Clin Invest 110: 1389-1398, 2002.

28. Ron D and Walter P: Signal integration in the endoplasmic reticulum unfolded protein response. Nat Rev Mol Cell Biol 8: 519-529, 2007.

29. Liu H, Miller E, van de Water B and Stevens JL: Endoplasmic reticulum stress proteins block oxidant-induced $\mathrm{Ca}^{2+}$ increases and cell death. J Biol Chem 273: 12858-12862, 1998.

30. Morris JA, Dorner AJ, Edwards CA, Hendershot LM and Kaufman RJ: Immunoglobulin binding protein (BiP) function is required to protect cells from endoplasmic reticulum stress but is not required for the secretion of selective proteins. J Biol Chem 272: 4327-4334, 1997.

31. Werstuck GH, Lentz SR, Dayal S, et al: Homocysteine-induced endoplasmic reticulum stress causes dysregulation of the cholesterol and triglyceride biosynthetic pathways. J Clin Invest 107: 1263-1273, 2001.

32. Ma Y and Hendershot LM: The role of the unfolded protein response in tumour development: friend or foe? Nat Rev Cancer 4: 966-977, 2004.

33. Boyce M and Yuan J: Cellular response to endoplasmic reticulum stress: a matter of life or death. Cell Death Differ 13: 363-373, 2006.

34. Li J and Lee AS: Stress induction of GRP78/BiP and its role in cancer. Curr Mol Med 6: 45-54, 2006.

35. Castro-Rivera E, Ran S, Thorpe P and Minna JD: Semaphorin 3B (SEMA3B) induces apoptosis in lung and breast cancer, whereas VEGF165 antagonizes this effect. Proc Natl Acad Sci USA 101: 11432-11437, 2004.

36. Pidgeon GP, Barr MP, Harmey JH, Foley DA and BouchierHayes DJ: Vascular endothelial growth factor (VEGF) up-regulates BCL-2 and inhibits apoptosis in human and murine mammary adenocarcinoma cells. Br J Cancer 85: 273-278, 2001.

37. Park SW, Kim HS, Hah JH, Kim KH, Heo DS and Sung MW: Differential effects between cyclooxygenase-2 inhibitors and siRNA on vascular endothelial growth factor production in head and neck squamous cell carcinoma cell lines. Head Neck 32: $1534-1543,2010$

38. Dal Monte M, Ristori C, Cammalleri M and Bagnoli P: Effects of somatostatin analogues on retinal angiogenesis in a mouse model of oxygen-induced retinopathy: involvement of the somatostatin receptor subtype 2. Invest Ophthalmol Vis Sci 50: 3596-3606, 2009

39. Wang L, Chen W, Xie X, He Y and Bai X: Celecoxib inhibits tumor growth and angiogenesis in an orthotopic implantation tumor model of human colon cancer. Exp Oncol 30: 42-51, 2008.

40. Abdelrahim M and Safe S: Cyclooxygenase-2 inhibitors decrease vascular endothelial growth factor expression in colon cancer cells by enhanced degradation of $\mathrm{Sp} 1$ and $\mathrm{Sp} 4$ proteins. Mol Pharmacol 68: 317-329, 2005. 